\title{
Clinical comparative analysis of monophasic and multiphasic acute disseminated encephalomyelitis in adults
}

Wei-Zhou Zang', Hong Yang ${ }^{1,2}$, Dan Li' ${ }^{1}$, Zheng-Da Zhao' ${ }^{1}$, Ya-Jing Sun ${ }^{1}$, Ming-Rong Xia', Shan Jiang ${ }^{1}$, Jie-Wen Zhang ${ }^{1}$

\begin{abstract}
1Department of Neurology, Henan Provincial People's Hospital, Zhengzhou, China 2Department of Neurology, Institute of Graduate School, Xinxiang Medical College, Xinxiang, China
\end{abstract}

Submitted: 3 March 2019

Accepted: 6 September 2019

Arch Med Sci

DOI: https://doi.org/10.5114/aoms.2020.99801

Copyright (c) 2020 Termedia \& Banach

\begin{abstract}
Introduction: This study aims to explore the clinical features and prognostic factors for relapse of acute disseminated encephalomyelitis (ADEM) in adults. Material and methods: 56 patients with ADEM were retrospectively analyzed. The epidemiological characteristics, clinical manifestations, laboratory features, magnetic resonance imaging (MRI), treatment and prognosis data of these patients were analyzed using the $\chi^{2}$ test for categorical variables and Mann-Whitney $U$-test for continuous variables. Then, the clinical characteristics and recurrence factors were summarized.

Results: 56 patients with ADEM, based on the criteria of the International Pediatric Multiple Sclerosis Study Group, were recruited to the study. Among these patients, 31 were male and 25 were female. Furthermore, 13 patients had multiphasic ADEM, and 29 patients (52\%) had definite incentive factors before onset. The commonest presenting symptoms and signs were fever $(36 \%)$, disturbance of consciousness (52\%), mental disorder (38\%), seizure $(14 \%)$, headache and dizziness (43\%), optic neuritis (34\%), autonomic nervous system symptoms (43\%), limb paralysis or abnormal sensation (73\%), and unilateral or bilateral pyramidal tract signs (48\%). Inflammatory changes in the cerebrospinal fluid were prominent. MRI T2-weighted and fluidattenuated inversion recovery images displayed multiple or large flaky high signals, and the lesions were usually different in the number and distribution of these lesions. Intravenous corticosteroids and/or immunoglobulin were still important treatments in the acute phase. After treatment, 38 patients completely recovered, 9 patients had neurologic deficits, and 9 patients died. Conclusions: ADEM in adults is not uncommon, its clinical features are complex and varied, and some of these are multiphasic. There may be some potential clinical predictors at first onset.
\end{abstract}

Key words: acute disseminated encephalomyelitis, adult, clinical characteristics, recurrence factors.

\section{Introduction}

Acute disseminated encephalomyelitis (ADEM) is a rare immune-related inflammatory demyelinating disease of the central nervous system (CNS). Its susceptible populations are children and adolescents. The clinical manifestations are new multifocal neurological abnormalities, and most of these

\author{
Corresponding author: \\ Jie-Wen Zhang \\ Department of Neurology \\ Henan Provincial People's \\ Hospital \\ No. 7 Weiwu Road \\ Jinshui District \\ Zhengzhou, Henan 450003, \\ China \\ Phone: +86 013607679900 \\ Fax: +86 0371-65580014 \\ E-mail: \\ zhangjw36201548@163.com
}


patients are complicated with encephalopathy (abnormal behavior or disturbance of consciousness).

Acute disseminated encephalomyelitis is usually considered as a monophasic disease. However, some case cohort studies have suggested that $25-33 \%$ of ADEM patients have recurrence. Furthermore, at the onset of this disease, it is impossible to predict which cases will recur. Most of the large cohort studies at home and abroad have focused on the first demyelinating episode in children, while few studies have been conducted on adult ADEM. In the present study, the clinical features of ADEM in adults were analyzed, in terms of epidemiology, clinical manifestations, laboratory examinations, neuroelectrophysiology, neuroimaging, treatment and prognosis. The aim of this study was to determine clinical features and predictive factors in adult patients with ADEM. Our hypothesis was that there are some epidemiological, clinical and laboratory biomarkers that might be associated with the treatment and prognosis of ADEM in adults.

\section{Material and methods}

\section{Study subjects}

A total of 73 patients with ADEM, who visited the Department of Neurology of Henan People's Hospital for the first time in the period from June 2015 to June 2017, were collected as subjects for observation. Since there are no unified diagnostic criteria for adult ADEM, and it was reported that the clinical manifestations of ADEM in adults and children are similar, in the present study, all patients were reevaluated by an experienced clinical deputy chief physician and an experienced radiologist, according to the diagnostic criteria established by the International Pediatric Multiple Sclerosis Research Group in 2012 [1, 2]. Encephalopathy was defined as altered consciousness or evident change of behavior at the time of attack onset not related to seizures or antiepileptic treatment. A diagnosis of multiple sclerosis (MS) was made when 2 non-ADEM demyelinating episodes subsequently occurred at least 3 months after the initial attack, fulfilling the McDonald criteria for dissemination in time and space [2].

Patients were excluded if another cause of neurological symptoms was suspected, including primary infectious, metabolic, toxic or systemic immunological causes. A total of 56 patients were finally included. After discharge, these patients received telephone or outpatient follow-ups from 3 months to 2 years, the median follow-up period being 1.6 years. According to whether new ADEM events occurred within 3 months after the first ADEM event or within the month after the complete hormone therapy, patients were divided into mono- phasic and multiphasic types. Monophasic ADEM was defined as a relapse-free interval of at least 2 years after the initial event. Multiphasic disease was defined as 2 episodes consistent with ADEM separated by 3 months but not followed by any further events [2]. The emergence of either new or old neurologic symptoms or lesions on magnetic resonance imaging (MRI) was defined as a flare if it occurred < 3 months after disease onset. A flare alone was not considered as multiphasic ADEM.

\section{Research methods}

The medical history, laboratory examination, imaging findings, treatment and follow-up data of these 56 patients were comprehensively analyzed.

Their medical history included the following items: general condition, clinical symptoms and signs.

The laboratory examination included the following items: routine blood test, C-reactive protein, liver function, kidney function, electrolyte, myocardial zymogram, glycosylated hemoglobin, 7 items of thyroid function, tumor markers, 3 items of rheumatoid, full set of immunity, connective tissue disease-related antibodies, virus antibody series (CMV, RV, TOX, HSV1 and HSV2), cerebrospinal fluid pressure, routine, biochemical and exfoliative cytologies, acid-fast and ink staining, oligoclonal bands, 6 items of encephalitis (anti-NMDAR, anti-CASPR2, anti-APMA1R, anti-LGI1R, anti-AMPA2R and anti-GABAbR), paraneoplastic antibodies (anti-amphiphysin, anti-CV2, anti-Ma2, anti-Ri, anti-Yo and anti-Hu), and AQP4.

Head and spinal cord MRI was performed [3]. The treatment method and interval from onset to high dose of glucocorticoid or immunoglobulin treatment was determined.

\section{Statistical analysis}

All data were statistically analyzed using SPSS 19.0. All count data (percentage) were evaluated using the 2 independent samples $\chi^{2}$ test or $\mathrm{R} \times \mathrm{C} t \chi^{2}$ test. The measurement data were evaluated using the 2 independent samples Mann-Whitney $U$-test. $P<0.05$ was considered statistically significant.

\section{Results}

\section{Demographic characteristics}

Among these 56 patients, 31 were male and 25 were female, the age of onset of the disease ranged within 18-76 years, and the average age of onset was 44.43 years. The difference in age of onset between monophasic and multiphasic ADEM was not statistically significant, multiphasic ADEM is more common in women, and the difference between the 2 groups was statistically significant (Table I). 
Table I. Comparison of clinical characteristics between monophasic and multiphasic acute disseminated encephalomyelitis

\begin{tabular}{|c|c|c|c|c|c|}
\hline Items & All patients & $\begin{array}{c}\text { Patients } \\
\text { with multiphasic } \\
\text { ADEM }\end{array}$ & $\begin{array}{c}\text { Patients } \\
\text { with monophasic } \\
\text { ADEM }\end{array}$ & $\begin{array}{l}\text { Statistical } \\
\text { value }\end{array}$ & $P$ value \\
\hline Sex (male/female) & $31 / 25$ & $3 / 10$ & $28 / 15$ & $\chi^{2}=7.139$ & 0.004 \\
\hline Age (years) ${ }^{b}$ & $\begin{array}{l}45.50 \\
(18 \sim 78)\end{array}$ & $\begin{array}{c}45.00 \\
(18 \sim 76)\end{array}$ & $\begin{array}{c}45.00 \\
(20 \sim 64)\end{array}$ & $U=276.0$ & 0.473 \\
\hline Inducement, $n(\%)$ & $29(51.79)$ & $5(38.46)$ & $24(55.81)$ & $\chi^{2}=1.204$ & 0.137 \\
\hline Fever, $n(\%)^{a}$ & $20(35.71)$ & $2(15.38)$ & $18(41.86)$ & $\chi^{2}=2.048$ & 0.079 \\
\hline Headache and dizziness, $n(\%)$ & $24(42.86)$ & $7(53.85)$ & $17(39.53)$ & $\chi^{2}=0.835$ & 0.181 \\
\hline Consciousness changes, $n(\%)$ & $29(51.79)$ & $8(61.54)$ & $21(48.84)$ & $\chi^{2}=0.645$ & 0.211 \\
\hline $\begin{array}{l}\text { Mental and behavioral } \\
\text { changes, } n(\%)^{a}\end{array}$ & $21(37.50)$ & $5(38.46)$ & $16(37.21)$ & $\chi^{2}=0.000$ & 0.500 \\
\hline Epilepsy, $n(\%)^{a}$ & $8(14.29)$ & $2(15.38)$ & $6(13.95)$ & $\chi^{2}=0.000$ & 0.500 \\
\hline $\begin{array}{l}\text { Optic neurological } \\
\text { symptoms, } n(\%)^{\mathrm{b}}\end{array}$ & 19 (33.93) & $8(61.54)$ & $11(25.58)$ & $\chi=4.265$ & 0.039 \\
\hline $\begin{array}{l}\text { Limb paralysis or abnormal } \\
\text { sensation, } n(\%)^{\text {a }}\end{array}$ & $41(73.21)$ & $10(76.92)$ & $31(72.09)$ & $\chi^{2}=0.000$ & 0.500 \\
\hline $\begin{array}{l}\text { Autonomic neurological } \\
\text { symptoms, } n(\%)\end{array}$ & $29(51.79)$ & $11(84.62)$ & $18(41.86)$ & $\chi^{2}=7.308$ & 0.004 \\
\hline $\begin{array}{l}\text { Unilateral or bilateral } \\
\text { pyramidal tract, } n(\%)\end{array}$ & $27(48.21)$ & $7(53.85)$ & $20(46.51)$ & $\chi^{2}=0.215$ & 0.322 \\
\hline $\begin{array}{l}\text { Number of days from } \\
\text { onset to the first treatment }{ }^{b}\end{array}$ & $6(1 \sim 24)$ & $13(6 \sim 24)$ & $6(1 \sim 21)$ & $U=59.000$ & 0.000 \\
\hline \multicolumn{6}{|l|}{ Laboratory examination } \\
\hline Leucocyte $\left(\times 10^{9} / \mathrm{l}\right)^{\mathrm{b}}$ & 9.38 (4.14 22.81) & $9.20(4.14 \sim 21.28)$ & 9.91 (4.18 22.81) & $U=259.50$ & 0.349 \\
\hline Neutrophil (\%) & $80.15(54.60 \sim 94.10)$ & $80.21(63.40 \sim 94.10)$ & 81.2 (54.60 93.79) & $U=266.00$ & 0.397 \\
\hline C-reactive protein $\left(\mathrm{mg} / \mathrm{l}^{\mathrm{b}}\right.$ & $13.00(0.10 \sim 87.12)$ & $14(0.90 \sim 37.60)$ & $13.20(0.10 \sim 87.12)$ & $U=228.00$ & 0.159 \\
\hline $\begin{array}{l}\text { Ventricle-cerebrospinal } \\
\text { fluid pressure }(\mathrm{mmHg})^{\mathrm{b}}\end{array}$ & $152.50(65 \sim 380)$ & 180 (65 210) & 145 (79 380) & $U=257.50$ & 0.335 \\
\hline $\begin{array}{l}\text { Cerebrospinal fluid } \\
\text { protein }\left(\mathrm{g} / \mathrm{l}^{\mathrm{b}}\right.\end{array}$ & $0.73(0.30 \sim 3.37)$ & $0.59(0.30 \sim 1.78)$ & $0.78(0.33 \sim 3.37)$ & $U=209.00$ & 0.086 \\
\hline $\begin{array}{l}\text { Cerebrospinal fluid } \\
\text { leucocytes }\left(\times 10^{6} / /\right)^{b}\end{array}$ & 18.5 (1 408) & $20(1 \sim 80)$ & $13(2 \sim 408)$ & $U=2715.0$ & 0.439 \\
\hline $\begin{array}{l}\text { Positive oligoclonal } \\
\text { bands of cerebrospinal } \\
\text { fluid, } n(\%)^{a}\end{array}$ & $11(19.64)$ & $6(46.15)$ & $5(11.63)$ & $\chi^{2}=5.510$ & 0.009 \\
\hline
\end{tabular}

ADEM - acute disseminated encephalomyelitis. Superscript "a" indicates that theoretical frequency of a cell was < 5, and continuous correction $\chi^{2}$ test was used. Data with a superscript "b" are non-normally distributed, and are expressed as median (minimum-maximum).

\section{Clinical characteristics}

Twenty-nine patients (52\%) had incentives of respiratory tract infection (15 patients), digestive tract infection (10 patients), drugs (2 patients) and vaccination (2 patients). In these 56 patients, fever occurred in 20 patients (36\%), consciousness changes occurred in 29 patients (52\%), mental and behavioral changes occurred in 21 patients (38\%), and epilepsy-like seizures occurred in 8 patients (14\%). Furthermore, headache and dizziness occurred in 24 patients (43\%), optic neurological symptoms occurred in 19 patients (34\%), limb paralysis or abnormal sensation occurred in 41 patients (73\%), autonomic neurological symp- toms occurred in 29 patients (52\%) and unilateral or bilateral pyramidal tract symptoms occurred in 27 patients (48\%). The differences in clinical manifestations between monophasic and multiphasic ADEM patients were not statistically significant, and most of these manifested as encephalopathy (abnormal mental behavior and change in consciousness) and multi-site injuries. However, optic and autonomic nerve symptoms were more common in multiphasic ADEM patients (Table I).

\section{Laboratory examination}

No significant abnormalities were found in the routine indexes (liver function, kidney function, 
electrolyte, myocardial zymogram, and glycosylated hemoglobin) of these 56 patients. Furthermore, 19 patients (34\%) had abnormal single or multiple autoimmune antibody indexes (thyroid antibody, ANCA, ENA and anti-nuclear antibody), 23 patients had abnormal viral antibody indexes, and 7 patients had abnormal IgM antibody. The routine blood test, CRP and routine cerebrospinal fluid test revealed prominent biochemical inflammatory changes. No positive results were found in exfoliative cytology, acid-fast and ink staining, 6 items of encephalitis, paraneoplastic antibodies and AQP4 in the cerebrospinal fluid in all patients. Lumbar puncture pressure increased in 20 patients (36\%). The oligoclonal bands in the cerebrospinal fluid were positive in 11 patients. The variable with a statistically significant difference between these 2 groups was the positive rate of the oligoclonal bands (Table I). Among the 6 multiphasic patients with positive oligoclonal bands, all oligoclonal bands in reexamination were negative when these recurred.

\section{Distribution characteristics of lesions in magnetic resonance imaging}

Magnetic resonance imaging revealed that lesions occurred in the brain parenchyma of all 56 patients. Lesions could also occur in the spinal cord of some patients. In the first attack, the lesions affected the cortical gray matter in 27 patients (48\%), the subcortical white matter in 29 patients (52\%), the cella lateralis in 21 patients (38\%), the corpus callosum in 11 patients (20\%), the deep gray matter in 27 patients (48\%), other supratentorial regions in 23 patients (41\%), the brain stem in 22 patients (39\%), the cerebellum in 13 patients (23\%), and the spinal cord in 17 patients (30\%). In multiphasic patients, the cortical gray matter and subcortical white matter were usually affected by lesions. Furthermore, supratentorial and subtentorial lesions coexisted, and the difference between these 2 groups was statistically significant (Table II).

\section{Treatment}

Glucocorticoid is the most commonly used drug in the acute stage of ADEM. All 56 patients in the acute stage were treated with high doses of methylprednisolone for shock treatment. The method was as follows: patients were intravenously dripped with $1,000 \mathrm{mg} /$ day of methylprednisolone for 3 days, shifted to an intravenous drip of $500 \mathrm{mg} /$ day of methylprednisolone for 3 days, and changed to an intravenous drip of $250 \mathrm{mg} /$ day of methylprednisolone for 3 days. Patients who achieved obvious relief of symptoms shifted to an oral use of $60 \mathrm{mg} /$ day of prednisone, which was decreased by $5 \mathrm{mg} /$ day every 2 weeks. Furthermore, 13 patients who achieved obvious relief of symptoms were given immunoglobulin, while 9 patients who achieved obvious relief of symptoms underwent shock therapy again. Six patients were treated with antiviral therapy according to the viral encephalitis in local hospitals.

\section{Follow-up results}

All 56 patients were followed up once every 3 months after discharge. Among these patients,

Table II. Comparison of magnetic resonance imaging characteristics between monophasic and multiphasic acute disseminated encephalomyelitis

\begin{tabular}{|c|c|c|c|c|c|}
\hline Parameter & $\begin{array}{c}\text { All patients, } \\
n(\%)\end{array}$ & $\begin{array}{c}\text { Patients with } \\
\text { multiphasic ADEM, } \\
n(\%)\end{array}$ & $\begin{array}{c}\text { Patients with } \\
\text { monophasic ADEM, } \\
n(\%)\end{array}$ & $\chi^{2}$ value & $P$ value \\
\hline \multicolumn{6}{|l|}{ Site of lesions } \\
\hline Cortical gray matter & $27(48.21)$ & $12(92.31)$ & $15(34.88)$ & 13.183 & 0.000 \\
\hline Subcortical white matter & $29(51.79)$ & $10(76.92)$ & $19(44.19)$ & 4.285 & 0.019 \\
\hline Cella lateralis ${ }^{a}$ & $21(37.50)$ & $5(38.46)$ & $16(37.21)$ & 0.000 & 0.500 \\
\hline Corpus callosuma & $11(19.64)$ & $1(7.69)$ & $10(23.26)$ & 0.704 & 0.201 \\
\hline Deep gray matter & $27(48.21)$ & $4(30.77)$ & $23(53.49)$ & 2.064 & 0.076 \\
\hline $\begin{array}{l}\text { Supratentorial and } \\
\text { subtentorial regions }\end{array}$ & $23(41.07)$ & $8(61.54)$ & $15(34.88)$ & 2.930 & 0.044 \\
\hline Brain stem & $22(39.29)$ & $6(46.15)$ & $16(37.21)$ & 0.335 & 0.282 \\
\hline Cerebellum $^{\mathrm{a}}$ & $13(23.21)$ & $2(15.38)$ & $11(25.58)$ & 0.151 & 0.349 \\
\hline Spinal cord ${ }^{a}$ & $17(30.36)$ & $5(38.46)$ & $12(27.91)$ & 0.145 & 0.352 \\
\hline \multicolumn{6}{|l|}{ Size of lesions } \\
\hline Small size $(<2 \mathrm{~cm})$ & $35(62.50)$ & $7(53.85)$ & $28(65.12)$ & 0.406 & 0.262 \\
\hline Big size $(\geq 2 \mathrm{~cm})$ & $21(37.50)$ & $6(46.15)$ & $15(34.88)$ & & \\
\hline
\end{tabular}

ADEM - acute disseminated encephalomyelitis. Superscript "a" indicates that the theoretical frequency of a cell was $<5$, and continuous correction $\chi^{2}$ test was used. 
21 patients were followed up in the Outpatient Department. At the end of follow-up, 43 patients who reached a follow-up time of at least 2 years presented with a monophasic course, while 13 patients presented with a multiphasic course. Among the multiphasic patients, 5 patients only presented with recurrence in time, while 8 patients presented with recurrence in time and space. For multiphasic patients, the first recurrence time was $6.9 \pm 5.3$ months, and the total recurrence number was $1.9 \pm 1.6$ times. In the multiphasic group, none of 13 patients had developed MS according to the diagnostic criteria.

Among monophasic patients, 25 patients (58\%) completely recovered, 9 patients (20\%) had neurological deficits, and 9 patients (20\%) died of severe infection. A total of 13 multiphasic patients in the acute stage were rehospitalized to receive a high dose of methylprednisolone, while 7 patients in the remission stage received mycophenolate mofetil to prevent recurrence. At the end of the last follow-up, 8 patients had neurological deficits, while 5 patients (all taking mycophenolate mofetil) completely recovered.

The outpatient follow-up of patients was reexamined by MRI. The result revealed that the lesions completely disappeared in 3 patients, were partially recovered in 11 patients, and continuously persisted in 4 patients. Three patients showed new lesions on MRI in this study. One patient showed a new lesion in the spinal cord 5 months after the first episode. One patient showed a new lesion in cortical gray matter 11 months after the first episode. Another patient showed a new lesion in the spinal cord and brain stem 10 months after the first episode. In addition, it was revealed that the course of the multiphase disease was correlated with the number of days from onset to the first treatment, and the difference between these 2 groups was statistically significant (Table I).

\section{Discussion}

In this retrospective study of 56 patients initially diagnosed with ADEM, we obtained several findings of clinical relevance: (1) approximately one-quarter of adult ADEM patients presented with a multiphasic course, and (2) some features of the initial presentation may distinguish monophasic from multiphasic disease.

It has been previously considered that ADEM is rare in adults. However, in recent years, the proportion of adult ADEM patients has gradually increased in reports on ADEM. In 2010, Ketelslegers et al. reported 25 adult ADEM patients and 92 children with ADEM, among whom 61 patients were diagnosed with ADEM after the follow-up. Among these cases, 54 were monophasic type, while only 7 were multiphasic type [1]. In 2016,
Koelman et al. conducted a retrospective study of ADEM with the largest sample size at 4 academic medical centers in the United States, in which 106 adult patients and 122 children were included. After the follow-up, 156 patients were diagnosed with monophasic type, while 23 patients were diagnosed with multiphasic type [3]. In the present study, approximately one-quarter (13/53) of adult ADEM patients had multiphasic type. The proportion of patients with a multiphasic course in this study was higher than those in previous studies mentioned above.

At disease onset, it is difficult to distinguish between the monophasic or multiphasic course in patients with first onset, according to clinical characteristics. In 2007, Mikaeloff et al. reported that the risk factors for ADEM recurrence included optic neuritis, family history of demyelinating disease of the CNS, MRI findings in accordance with Barkhof's criteria for MS, and the absence of sequelae after the first onset of the disease [4]. Consistent with the results reported by Mikaeloff, the present retrospective study also revealed that some initial clinical characteristics could be used to distinguish between the monophasic and multiphasic course. For example, multiphasic patients often have symptoms of optic nerve and autonomic nerve damage. In addition, the recurrence of ADEM is more common in women, patients with transient positive oligoclonal bands, and patients with lesions in the cortical gray matter, subcortical white matter, and both supratentorial and infratentorial lesions revealed by MRI.

It has been reported previously that the maleto-female radio was $1.0: 0.8$ in patients with ADEM, the recurrence rate was $25-33 \%$, and recurrent patients were more likely to be female [1, 5]. The results of the present study are consistent with the above reports. Our results also revealed that adult ADEM was more common in 40-50 yearold patients, but rare in patients over 60 years old, and the average age of onset was 44.43 years old. This is not consistent with the average age of 33.5 years reported in the literature [6].

ADEM, also termed postinfectious encephalomyelitis, usually occurs after minor infectious illnesses. Unlike infectious encephalitis, the absence of viral or bacterial antigens within the CNS is nearly universal in ADEM. However, the laboratory indicators of ADEM lack specificity. In the present study, the inflammatory indexes in some patients increase, such as white blood cells and CRP, and this may be correlated with the pathogenesis of the disease (infection and immunity). In the cerebrospinal fluid examination, leukocyte count increased and protein level slightly increased in most patients, but normal results may occur. These are similar to those reported in the literature [7]. 
Although ADEM has been investigated for more than 200 years, most scholars have considered that oligoclonal bands are absent in ADEM patients, and used it as a basis for distinguishing ADEM from MS. However, more and more case follow-up studies have revealed that in a few cases (12.5\%), the oligo clonal bands were positive but, differently from MS, in ADEM they were transient [8]. In the present study, positive oligoclonal bands were detected in $19.64 \%$ of the patients, and these were transient in $10.71 \%$ of patients. Moreover, the present study also revealed that transient positive oligoclonal bands may be correlated with recurrence. This is similar to that reported in the literature [9]. More studies are needed to confirm this conclusion.

The most widely used diagnostic method of ADEM is MRI. MRI is also one of the tools to distinguish ADEM from other demyelinating diseases of the CNS. Studies have reported that $[7,10]$ on T2-weighted images or fluid attenuation reversal recovery (FLAIR) pulse sequence, the lesions of ADEM were characterized by multiple, patchy and unclear margins, and larger lesions $(>1-2 \mathrm{~cm})$, and these lesions often affected the subcortical and deep white matter, the gray-white matter junction area, the deep gray matter nuclei (bilateral thalamus, basal ganglia), the cerebellum and the brainstem, and up to $1 / 3$ of the spinal cord is affected. The present study also revealed that ADEM lesions presented with multiple or large patchy $\mathrm{T} 2$ and FLAIR high signal shadows, and the number and distribution of these lesions could greatly vary. Although the diagnostic criteria for MRI have not yet been established, a cohort study revealed that a $\mathrm{T} 1$ low signal ( $\mathrm{T} 1$ black hole) is common in MS, but is rare in ADEM. Furthermore, lenticular nucleocapsid involvement is more common in ADEM than in MS and neuromyelitis optica (NMO). Yang et al. reported that recurrence of ADEM was correlated with the coexistence of supratentorial and subtentorial lesions of the CNS, and the presence of spinal cord lesions, but it was not correlated with basal nuclei, semioval center, periventricular, and brainstem lesions [11]. But the present study revealed that recurrence may be correlated with the involvement of cortical gray matter and subcortical white matter, and the coexistence of supratentorial and subtentorial lesions. This is incompletely consistent with observations reported in the literature. The reason may be related to the research samples. That is, the subjects in studies conducted by Yang et al. were children, while the subjects in the present study were adults.

Although the development of MS after ADEM is considered rare, the differential diagnosis between multiphasic ADEM and MS is still important. The presence of encephalopathy at onset can facilitate the distinction between ADEM and MS, because encephalopathy is very unusual in MS [1]. In the present study, all of the 257 patients enrolled had symptoms of encephalopathy defined as mental status changes and/or behavioral alterations such as marked irritability, and none of them had progressed to MS according to the diagnostic criteria.

At present, there is no evidence from a clinically controlled study on drug treatment of ADEM. However, it has been generally considered that ADEM is an immune-related disease. In the acute stage, it is usually treated with intravenous injection of high doses of corticosteroids. When the curative effect is not good, it can be used in combination with immunoglobulin. It is possible that high doses of corticosteroids can inhibit immune function, stabilize lysosome membrane, reduce brain and spinal cord edema, and inhibit the inflammatory demyelinating course. Through active treatment, most patients have achieved good prognosis, some patients had neurological deficits, such as motor dysfunction and cognitive impairment, and few of the severe patients died of infections and untimely treatment. In particular, the present study revealed that the determination of whether corticosteroids can be intravenously injected in time in the acute stage was related to recurrence.

The limitations of this study include its retrospective nature and relatively low number of patients resulting from the single-center nature. In addition, the follow-up period in this study was short. Data from multiple centers are needed to confirm our findings.

\section{Acknowledgements}

Fund program Scientific and Technological Research Project of Henan Province in 2015 (152102 310140.0).

\section{Conflict of interest}

The authors declare no conflict of interest.

\section{References}

1. Ketelslegers IA, Visser IER, Neuteboom RF, Boon M, Catsman-Berrevoets CE, Hintzen RQ. Disease course and outcome of acute disseminated encephalomyelitis is more severe in adults than in children. Mult Scler 2011; 17: 441-8.

2. Krupp LB, Tardieu M, Amato MP, et al. International Pediatric Multiple Sclerosis Study Group. International Pediatric Multiple Sclerosis Study Group criteria for pediatric multiple sclerosis and immune-mediated central nervous system demyelinating disorders: revisions to the 2007 definitions. Mult Scler 2013; 19: 1261-7.

3. Koelman DLH, Chahin S, Mar SS, et al. Acute disseminated encephalomyelitis in 228 patients: a retrospec- 
tive, multicenter US study. Neurology 2016; 86: 2085-93.

4. Mikaeloff Y, Caridade G, Husson B, Suissa S, Tardieu M. Acute disseminated encephalomyelitis cohort study: prognostic factors for relapse. Eur J Paediatr Neurol 2007; 11: 90-5.

5. Tenembaum S, Chamoles N, Fejerman N. Acute disseminated encephalomyelitis: a long-term follow-up study of 84 pediatric patients. Neurology 2002; 59: 1224-31.

6. Schwarz S, Mohr A, Knauth M, Wildemann B, StorchHagenlocher B. Acute disseminated encephalomyelitis: a follow-up study of 40 adult patients. Neurology 2001; 56: 1313-8.

7. Hynson JL, Kornberg AJ, Coleman LT, Shield L, Harvey AS, Kean MJ. Clinical and neuroradiologic features of acute disseminated encephalomyelitis in children. Neurology 2001; 56: 1308-12.

8. Pohl D, Alper G, Van Haren K, et al. Acute disseminated encephalomyelitis: updates on an inflammatory CNS syndrome. Neurology 2016; 87: S38-45.

9. Suppiej A, Vittorini R, Fontanin M, et al. Acute disseminated encephalomyelit-is in children: focus on relapsing patients. Pediatr Neurol 2008; 39: 12-7.

10. Verhey LH, Branson HM, Shroff MM, et al. MRI parameters for prediction of multiple sclerosis diagnosis in children with acute CNS demyelination: aprospective national cohort study. Lancet Neurol 2011; 10: 1065-73.

11. Yang B, Jiang LL, Ye XF, et al. Clinical feature and prognostic factors for relapse of acute disseminated encephalomyelitis in children. Chin J Applied Clin Pediatr 2011; 26: 1019-21. 